\title{
Role of the striatum and the cerebellum in motor skill acquisition
}

\author{
Markus M. Schugens ${ }^{\mathrm{a}, *}$, Caterina Breitenstein ${ }^{\mathrm{b}}$, \\ Hermann Ackermann ${ }^{\mathrm{c}}$ and Irene Daum ${ }^{\mathrm{a}}$ \\ ${ }^{a}$ Neuropsychology, Faculty of Psychology, \\ Ruhr-University of Bochum, Germany \\ ${ }^{\mathrm{b}}$ Department of Neurology, University of Southern \\ California, USA \\ ${ }^{\mathrm{c}}$ Department of Neurology, University of Tübingen, \\ Germany
}

\begin{abstract}
Motor skill acquisition was investigated in patients with Parkinson's disease (PD) or cerebellar dysfunction using two sensory-guided tracking tasks. The subjects had to learn to track a visual target (a square) on a computer screen by moving a joystick under two different conditions. In the unreversed task, the horizontal target movements were semi-predictable and could be anticipated. In the reversed task, the horizontal movements of a pointer which had to be kept within the target square were mirror-reversed to the joystick movements. PD patients showed intact learning of the semipredictable task and reduced learning of the mirror-reversed task; patients with cerebellar dysfunction showed the opposite pattern. These findings are discussed in relation to the differential contribution of the cerebellum and the striatum to motor skill acquisition: the cerebellum appears to participate in the implementation of anticipatory movements, whereas the striatum may be critically involved in types of motor learning which require a high degree of internal elaboration.
\end{abstract}

Keywords: Motor skill acquisition, Parkinson's disease, cerebellar dysfunction

\section{Introduction}

Skill acquisition refers to the ability to learn through practice (see [6]). Motor skill learning is a typical example of non-declarative learning, i.e., behavioural changes as a result of experience that are not dependent upon explicit reference to previous training sessions or stored memory contents. Consistent with this view, patients with severe amnesia were unimpaired at acquiring motor skills in a range of tasks including mirror drawing or rotor pursuit (see [24]). Intact

\footnotetext{
* Corresponding author: Dr. Markus Schugens, Neuropsychology, Faculty of Psychology, Ruhr-University of Bochum, 44780 Bochum, Germany. E-mail: markus.m.schugens@ruhr-uni-bochum.de.
}

hippocampal-diencephalic circuitry therefore does not seem to be necessary for new motor learning.

The striatum and the cerebellum, on the other hand, appear to be critically implicated in the acquisition of motor skills, as suggested by theoretical accounts and empirical evidence from animal studies (e.g., $[16,18,19])$. Neuroimaging studies also confirmed that learning and automatisation of different motor skills are accompanied by significant blood flow changes in both brain areas, but the pattern of results is far from consistent [6]. There is, e.g., disagreement as to whether motor learning is associated with an increase or decrease of cerebellar activity or whether there is a shift from cerebellar to striatal and cortical activation from the early stages of learning to increasing automaticity. The findings of a particular study are dependent upon the specific features of the learning paradigm and the stage of acquisition at the time of scanning (for a discussion see [6]).

Evidence from neuropsychological investigations have also supported the hypothesis of a striatal and/or cerebellar mediation of motor learning. Patients suffering from Huntington's or Parkinson's disease (PD) were found to be impaired at learning different types of manual tracking tasks (e.g., $[8,10,11])$. Other studies, however, reported intact sensorimotor learning of PD patients on such tasks, and whether or not a deficit is observed may be contingent upon the severity of motor dysfunction which - in most cases - implies pathological changes outside the basal ganglia (for a summary see [15]). Patients with pathology limited to the cerebellum had problems in learning to trace a geometrical pattern in a skillful manner under normal visual guidance (but not under mirror-reversed conditions). This finding was attributed to deficient movement strategies [21].

Neuropsychological studies in which an identical or conceptually very similar learning task was administered to both patients with basal ganglia disorders and patients with cerebellar dysfunction are sparse. The findings available so far suggest that the two brain regions partly differ in their involvement in motor learning. A frequently used neuropsychological learning 
paradigm is the serial reaction time task (SRT) which requires the acquisition of a sequence of new sensorimotor associations between the spatial positions of stimuli and responses. In a direct comparison of both groups, patients with cerebellar degeneration showed a pronounced deficit in learning this task, whereas the impairment of PD patients was relatively mild [20]. Other authors, however, have reported a comparable impairment of both patient groups on the SRT task [7]. Factors such as sample differences in disease severity and the presence of cognitive deficits may partly account for such discrepant results, since PD patients with frontal signs were found to perform worse on the SRT task than patients with intact frontal function [14].

Clearer dissociations between the performance of PD patients and patients with cerebellar pathology were observed in a simple associative motor learning task, classical eyeblink conditioning. Patients with cerebellar pathology were unable to acquire conditioned motor responses, whereas conditioning was unimpaired in early PD in the same experimental procedure $[3,5]$.

The aim of this study was to further investigate the specific contribution of the cerebellum and the basal ganglia to motor learning, using two continuous visually-guided tracking tasks which differed with respect to movement predictability and demands on visuomotor integration. To our knowledge, acquisition of manual tracking has not yet been compared in PD patients and patients with cerebellar dysfunction. A particular issue of interest was to explore whether predictability and visuomotor demands, factors which are known to influence motor skill acquisition [15] differentially affected the learning curves of both patient groups.

\section{Method}

\subsection{Subjects}

A group of patients with idiopathic Parkinson's disease (PD) and a group of patients with cerebellar disorders (CERE) took part in this study. All patients were recruited from the outpatient clinic of the Department of Neurology, University of Tübingen. As the patient groups differed on sample size and the presence of left-handers, two separate healthy control groups were formed. For each patient group, a group of normal control subjects (NC) closely matched to the patients on background variables (sex, age, handedness) was selected from a larger pool of healthy volunteers recruited by advertisements. None of the controls had a history of psychiatric or neurological problems or took medication at the time of testing. The study was conducted with approval by the local ethics committee.

\subsection{Parkinson's disease patients and their control subjects}

The Parkinson's disease patients (group PD) included 9 men and 5 women in the earlier stages of the disease (average age $=53.1 \mathrm{yrs}, \mathrm{SD}=13.1$ ). Six patients were rated as grade I and 9 patients as grade II on the Hoehn and Yahr scale [12]. At the time of testing, 2 patients did not take any antiparkinsonian drugs. The remaining patients were on stable medication consisting of L-Dopa in combination with a MAO-B-inhibitor, a D2- agonist and/or a noncompetitive NMDA-antagonist. None of the patients had undergone neurosurgical operation or showed evidence of dementia in a comprehensive neuropsychological battery including tests of memory and executive function (see [4]). Patients who experienced fluctuations of motor capacities were tested at a time during the day when they subjectively felt that they had optimum motor functioning.

A group of 14 healthy volunteers ( 9 men, 5 women, group NC) also completed the tasks. Their mean age was 50.9 years $(\mathrm{SD}=11.4)$. All PD patients and control subjects were right-handed.

\subsection{Patients with cerebellar dysfunction and their control subjects}

The group of patients with cerebellar dysfunction (group CERE) comprised 4 men and 4 women (average age $=58.4$ years, $S D=15.0$ ). Five patients suffered from idiopathic cerebellar ataxia (ICA), one patient had a diagnosis of autosomal dominant cerebellar ataxia (ADCA). The ICA diagnosis was based on clinical signs of a purely cerebellar syndrome (stance or gait ataxia, oculomotor signs, dysmetria or intention tremor of the upper limbs and dysarthria) and MRI evidence of atrophy restricted to the cerebellum. The ADCA patient had a family history of cerebellar ataxia. The two remaining patients showed an ischemic lesion which involved the paravermal superior portions of the right cerebellum, as documented by MRI evidence. Two of the cerebellar ataxia patients were medicated with the serotonin precursor 5-hydroxytryptophan, the two ischemia patients were taking a low dosis of as- 
pirin. None of the CERE patients showed evidence of dementia (see [2]).

The comparison group consisted of 8 healthy volunteers ( 4 men, 4 women, group NC) with a mean age of 58.9 years $(\mathrm{SD}=9.5)$. One CERE patient and one NC subject were left-handed, all other subjects were right-handed.

\subsection{Tracking tasks}

Two tracking tasks were developed on the basis of a procedure described by Frith and coworkers [8]. The subjects were instructed to try to keep a pointer (a cross) within a moving target (a $7 \mathrm{~mm}$ square) which appeared on a computer screen. The position of the pointer was controlled by a small $3.5 \mathrm{~cm}$ joystick which the subject operated by his/her preferred hand. One $\mathrm{cm}$ movement of the joystick translated into $3.75 \mathrm{~cm}$ of pointer movement on the screen. The horizontal and vertical movements of the target were varied independently. The movement directions were determined every $25 \mathrm{msec}$ by the combination of sine waves.

Unreversed tracking task. In the unreversed tracking task, the horizontal movements were determined by a single sine wave of $0.15 \mathrm{~Hz}$ and were therefore regular and predictable. The vertical movements resulted from a combination of 3 different sine waves $(0.06 \mathrm{~Hz}$, $0.11 \mathrm{~Hz}, 0.23 \mathrm{~Hz}$ ) which resulted in an unpredictable movement.

The frequency of the horizontal movement was changed from $0.3 \mathrm{~Hz}$ in the Frith et al. [8] study to $0.15 \mathrm{~Hz}$ because in pilot investigations two patients with cerebellar disorders and two elderly PD patients had extreme difficulties with performing the task. This reduction in frequency made a later exclusion of subjects obsolete, since all participants were more than $5 \%$ of the time on target (see [8]).

Reversed tracking task. In the reversed tracking task, both horizontal and vertical movements were unpredictable and determined by the combination of 3 sine waves (horizontal: $0.03 \mathrm{~Hz}, 0.21 \mathrm{~Hz}, 0.32 \mathrm{~Hz}$; vertical: $0.05 \mathrm{~Hz}, 0.19 \mathrm{~Hz}, 0.33 \mathrm{~Hz}$ ). In addition, the direction between the movements of the joystick and the pointer were mirror-reversed in horizontal direction, i.e., joystick movements to the left resulted in pointer movements to the right on the screen and vice versa.

\subsection{Procedure}

At the beginning of the testing session, the subjects practiced controlling the position of the pointer on the screen by operating the joystick (without the target square being shown), until they stated that they were confident in controlling the pointer using the joystick. This was followed by 3 minutes of continuous practice of the unreversed tracking task and a 6 min rest. After the break, a second 3 min training session was performed. In accordance with the procedure used by Frith et al. [8], the participants were not given any information about the regularity of the horizontal movements in the unreversed task. After 20 min of unrelated tests, the subjects were instructed that the movements of the pointer on the screen and the joystick were mirror-reversed in the following tests, and the participants practiced the new pattern of control. They then completed two 3 min training sessions of the reversed task which were again separated by a 6 min rest. In a brief post-experimental interview the subjects were asked whether they had noticed anything special while performing the tasks.

Analysis. The two training sessions of the unreversed and the reversed tracking tasks were subdivided into 6 trials of $30 \mathrm{sec}$ each. For each trial, the total time for which the subject was able to keep the pointer within the target limits (i.e., the $7 \times 7 \mathrm{~mm}$ square) was determined (in \%). All participants fulfilled the criterion that their performance was 'on target' in the horizontal and vertical directions for more than $5 \%$ of the time (see [8]), therefore the data of all subjects entered analysis.

In accordance with the procedure used by Frith and coworkers [8], the difference between the time-ontarget scores during the first two $30 \mathrm{sec}$ trials of each session were analysed as a measure of the acquisition of a motor set. Set acquisition is thought to reflect the short-term adaptation of a motor program to the specific requirements of a task (see [8]). For both tracking tasks, set acquisition was analysed by repeated measures ANOVA with the factors Group (PD or CERE vs. their respective NC), Session (1 vs. 2) and Trial (first vs. second $30 \mathrm{sec}$ interval).

Improvements of the time-on-target scores during the course of trials 2 to 6 within each session have been interpreted as temporary learning, whereas betweensession improvements are thought to reflect more permanent learning and increasing automatisation [8]. These two aspects of motor learning were analysed by repeated measures ANOVA with the factors Group (PD or CERE vs. their respective NC), Session (1 vs. 2) and Trials (second to sixth $30 \mathrm{sec}$ interval, linear trend). 


\section{Results}

\subsection{Skill acquisition in Parkinson's disease patients}

Unreversed tracking task. The time-on-target data for the PD patients and their NC subjects are shown in Fig. 1, separately for the predictable horizontal and the unpredictable vertical directions.

In all analyses described in the following, significant main Group effects were observed (all $p<0.01$ ) which indicate that the PD patients generally had more problems in performing the tracking tasks than the controls. The main Group effects are not separately described for each ANOVA.

Analysis of adaptation for the predictable horizontal direction yielded a significant interaction (Group $\times$ Session $\times$ Trial, $F(1,26)=9.12, p=0.006)$. The NC subjects showed a large improvement within the first minute of the first session, whereas the scores of the PD patients decreased; there were no group differences at the beginning of the second session. For the unpredictable vertical direction, the controls also showed significant improvements within the first minute of tracking, whereas there were no significant changes in the PD patients (Group $\times$ Trial, $F(1,26)=6.80$, $p=0.015)$.

Significant learning was observed within and between sessions for both the horizontal and vertical directions (Trial (linear trend) and Session, all $F(1,26)>8.36$, all $p<0.009)$. There were no significant interactions involving the Group factor, i.e., no significant group differences with respect to measures of temporary and permanent learning. The PD patients alone showed significant motor skill acquisition in terms of significant between-session improvements in tracking performance both for the predictable horizontal $(p<0.001)$ and the unpredictable vertical direction $(p=0.007)$.

Reversed tracking task. The tracking data for the reversed tracking task are depicted in Fig. 2, separately for the mirror-reversed horizontal and the unreversed vertical direction.

The NC group tended to increase their time-ontarget scores within the first minute of mirror-reversed tracking, whereas the PD patients tended to decrease their scores (Group $\times$ Trial, $F(1,26)=7.98, p=$ $0.009)$; there were no significant group differences for the vertical direction.

Significant learning effects emerged in the analysis of the tracking data both within and between sessions
(Trial and Session, all $F(1,26)>8.55, p<0.008$ ). However, the improvements of the tracking scores from the first to the second session were significantly larger in the NC group compared to the PD patients, for both the mirror-reversed horizontal and the unreversed vertical components (Group $\times$ Session, both $F(1,26)>9.79, p<0.005)$. Analysis of temporary within-session learning did not yield significant group differences. The PD patients alone did not show significant skill acquisition in terms of betweensession improvements, neither for the reversed horizontal ( $p=0.16$ ) nor for the unreversed vertical direction $(p=0.21)$.

Two PD patients (14.3\%) and 3 NC subjects (21.4\%) reported in the post-experimental interview that they had noticed that the horizontal movement of the target was regular and predictable when they practiced the unreversed task. The proportion of subjects who had gained insight into features of the task which were relevant for behavioural control thus did not differ between the two groups.

\subsection{Skill acquisition in patients with cerebellar dysfunction}

Unreversed tracking task. The tracking data of the CERE patients and their respective control subjects are illustrated in Fig. 3, separately for the predictable horizontal and the unpredictable vertical directions.

With the exception of the horizontal direction of the mirror-reversed task which yielded only a tendency towards group differences in overall time-on-target $(p=0.094)$, all other comparisons described in the following yielded significant main Group effects (all $p<0.035$ ) which indicates a general motor execution deficit in patients with cerebellar dysfunction. These main effects are not separately discussed for each analysis.

Analysis of the tracking scores for the first minute of each session yielded significant improvements from the first to the second $30 \mathrm{sec}$ trial for the horizontal direction (Trial, $F(1,14)=7.18, p=0.018$ ), the scores for the vertical direction did not reach significance. There were no significant group differences for this measure of short-term adaptation.

For the predictable horizontal direction, the timeon-target scores improved significantly within each session and from the first to the second session (Trial and Session, both $F(1,14)>6.40, p<0.025)$. The CERE patients, however, showed a smaller improvement over sessions than the NC subjects (Group $\times$ Session, $F(1,14)=5.55, p=0.034)$, whereas anal- 
horizontal (predictable)

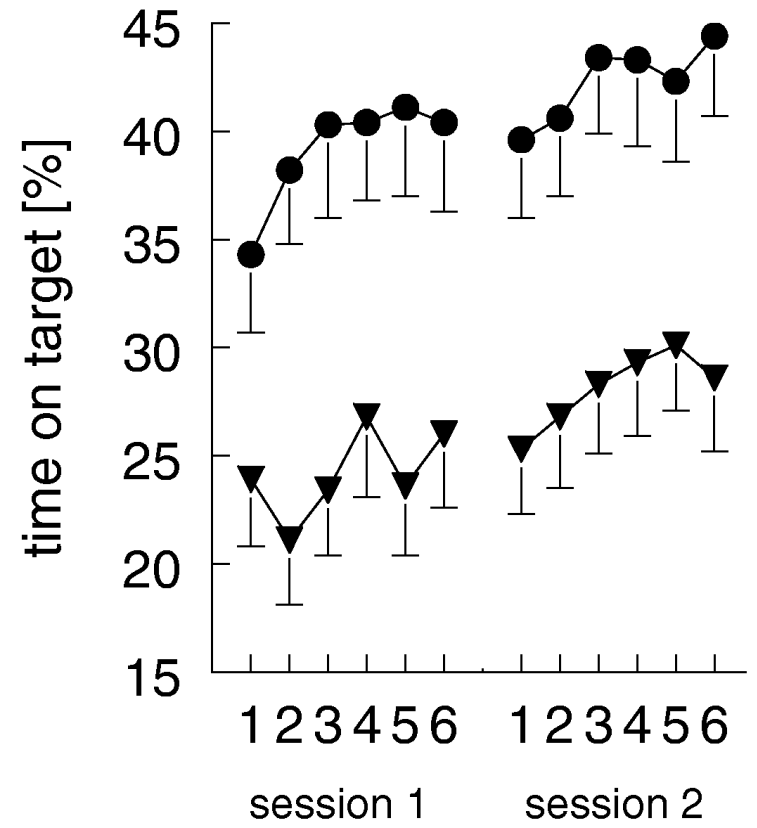

vertical (unpredictable)

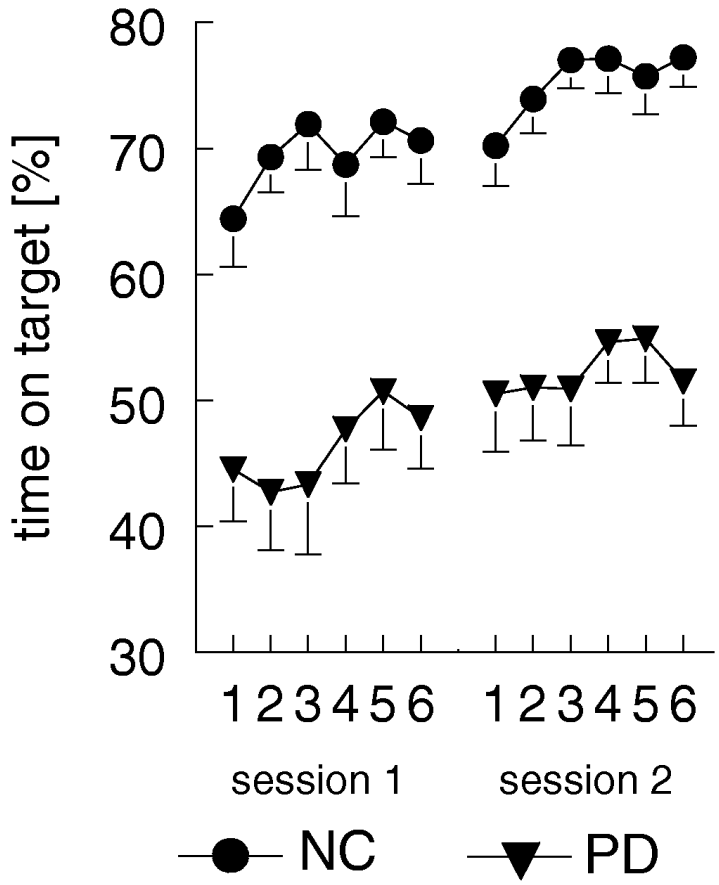

Fig. 1. Mean time on target (and SEM) for the predictable horizontal component (upper graph) and the unpredictable vertical component (lower graph) of the unreversed manual tracking task ( $\mathrm{PD}=$ Parkinson's disease patients; $\mathrm{NC}=$ control subjects). horizontal (mirror-reversed)
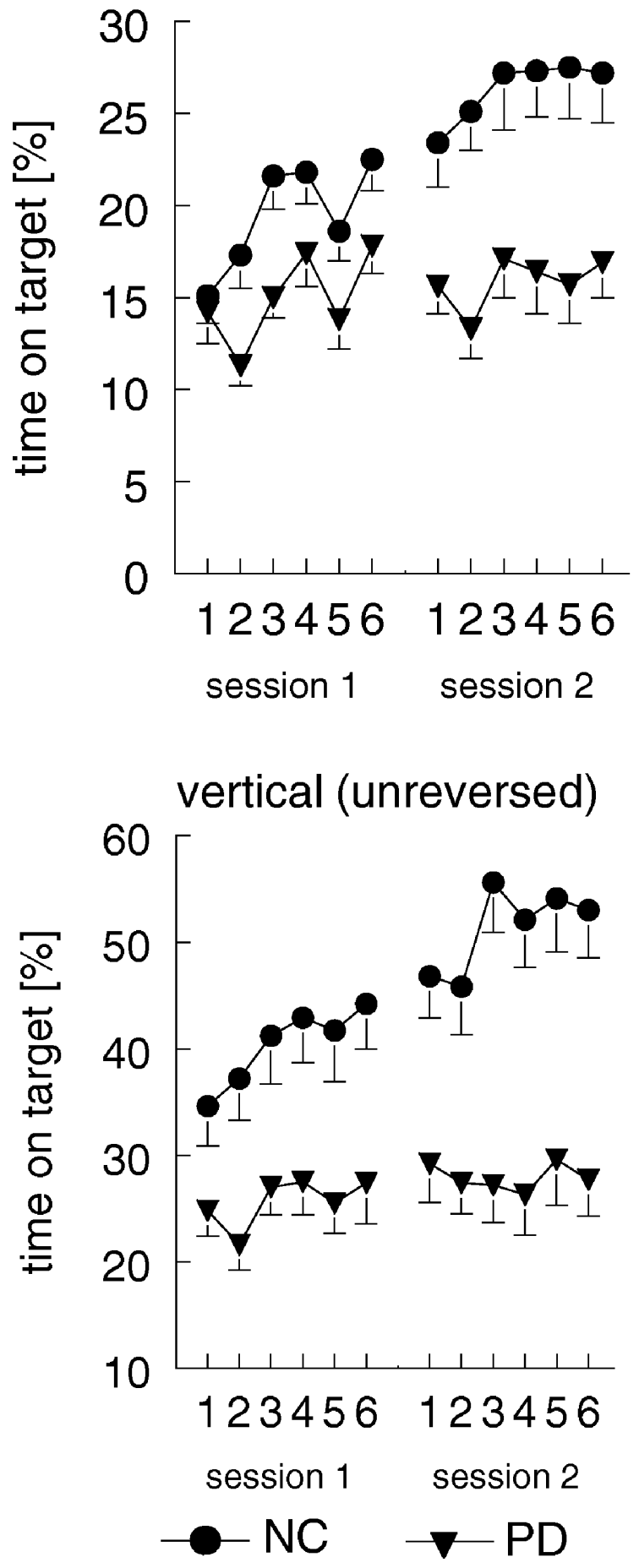

Fig. 2. Mean time on target (and SEM) for the mirror-reversed horizontal component (upper graph) and the unreversed vertical component (lower graph) of the reversed manual tracking task ( $\mathrm{PD}=$ Parkinson's disease patients; $\mathrm{NC}=$ control subjects $)$. 
horizontal (predictable)
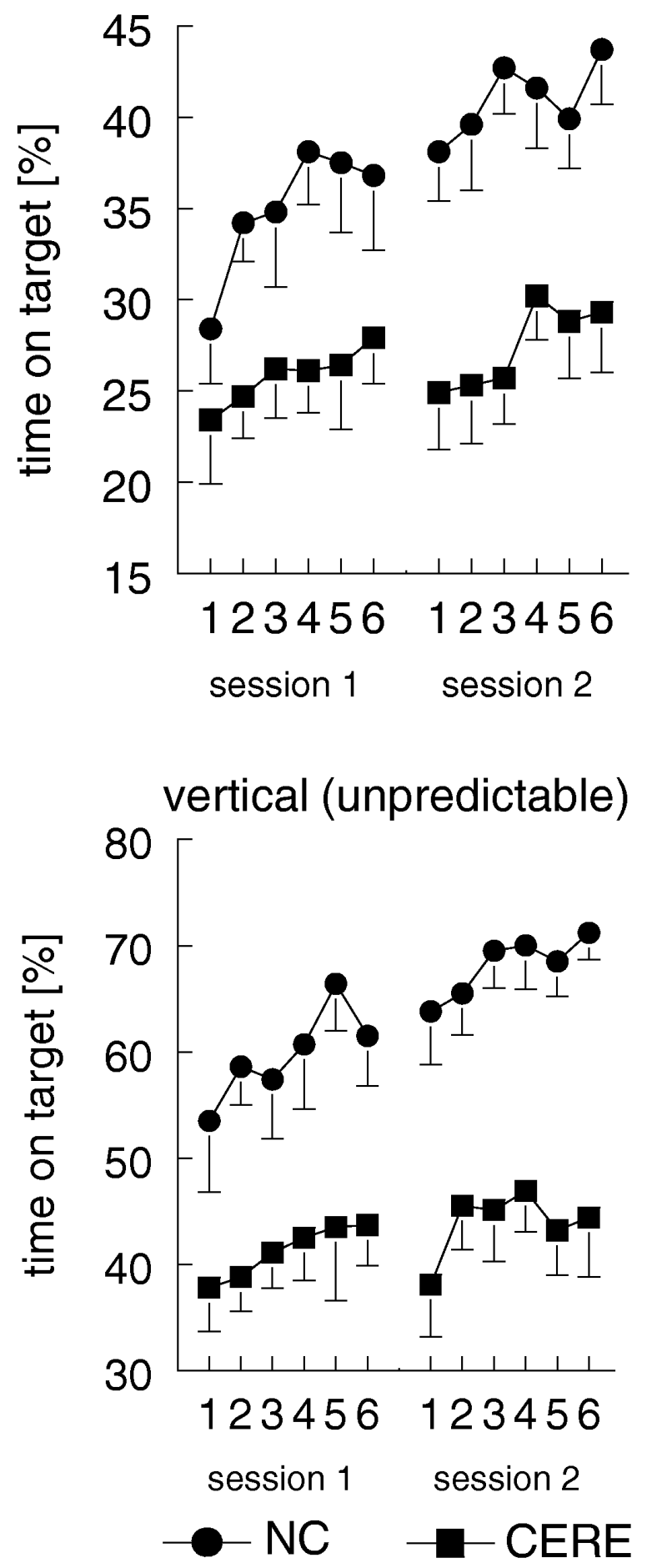

Fig. 3. Mean time on target (and SEM) for the predictable horizontal component (upper graph) and the unpredictable vertical component (lower graph) of the unreversed manual tracking task (CERE = patients with cerebellar dysfunction; $\mathrm{NC}=$ control subjects). horizontal (mirror-reversed)
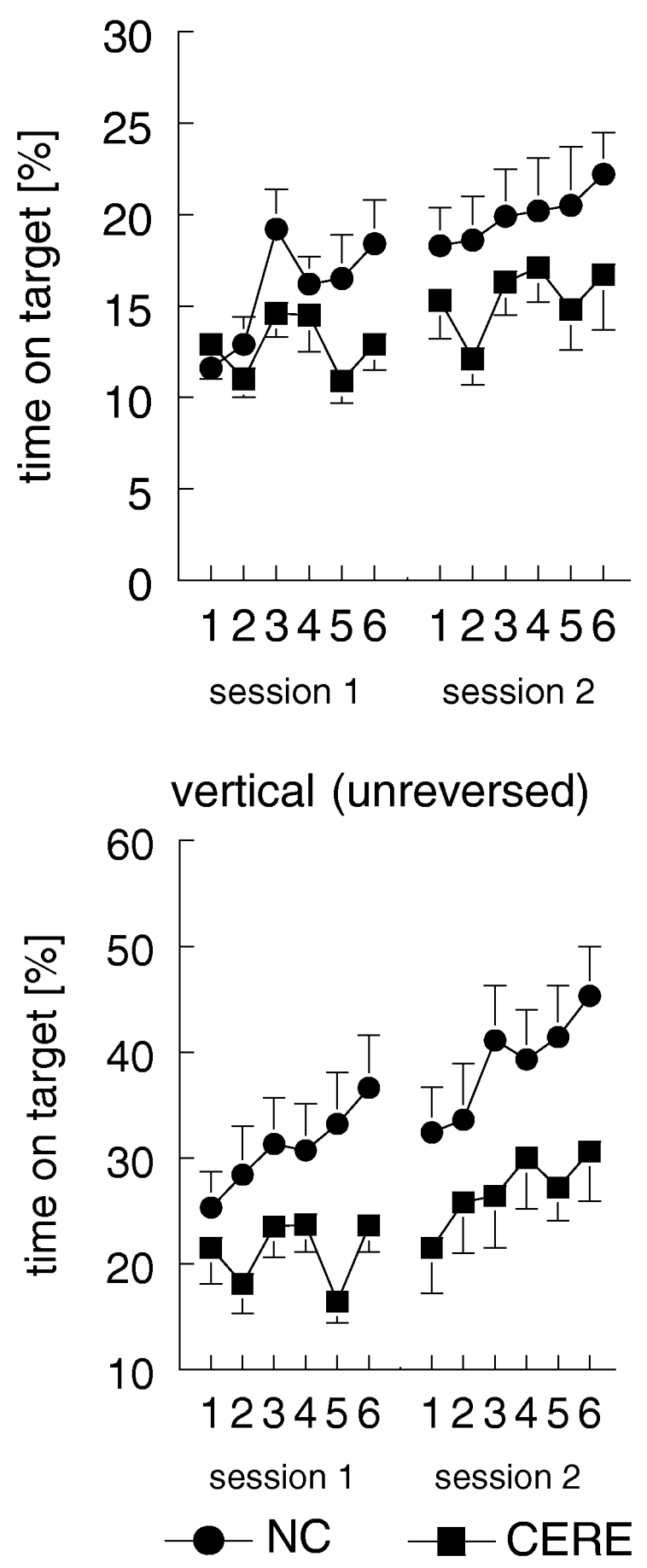

Fig. 4. Mean time on target (and SEM) for the mirror-reversed horizontal component (upper graph) and the unreversed vertical component (lower graph) of the reversed manual tracking task $(\mathrm{CERE}=$ patients with cerebellar dysfunction; $\mathrm{NC}=$ control subjects). 
ysis of the temporary within-session effects did not yield a significant group difference.

The tracking data for the vertical direction indicated significant learning across sessions (Session, $F(1,14)=13.65, p=0.002)$; the within-session changes after initial adaptation did, however, not reach significance (linear trend: $p=0.12$ ). None of the interactions involving measures of temporary or more permanent learning and the Group factor reached significance.

Analysis of the data of the CERE group alone indicated that there was no significant motor skill learning on the unreversed task, since there was no significant between-session improvement in tracking performance either for the predictable horizontal $(p=0.16)$ or for the unpredictable vertical direction $(p=0.21)$.

Reversed tracking task. The tracking data for the reversed tracking condition are illustrated in Fig. 4, separately for the mirror-reversed horizontal and the unreversed vertical target movements.

There were no significant changes for the time-ontarget scores between the first and the second $30 \mathrm{sec}$ trials either for the mirror-reversed or the vertical direction.

For both directions, the time-on-target scores improved significantly both within and between sessions (Trial and Session, both $F(1,14)>9.67, p<0.009$ ). None of the interactions involving the Group factor reached significance, i.e., the CERE and the NC groups did not differ with respect to the temporary and more permanent learning effects.

Analysis of the learning curves of the CERE patients alone indicated that there was evidence for motor skill acquisition on the mirror-reversed task: the between-session effects were near-significant both for the reversed horizontal component $(p=0.055)$ and the unreversed vertical component $(p=0.055)$.

Two CERE patients (25\%) and two NC subjects $(25 \%)$ reported during the post-experimental interview that they had noticed that the horizontal target movements were regular and predictable when they practiced the unreversed task. The proportion of subjects who had gained declarative knowledge about task-relevant information was thus identical in the two groups.

\section{Discussion}

The aim of this study was to investigate the differential involvement of the striatum and the cerebellum in motor skill acquisition by comparing the learning curves of PD patients and patients with cerebellar dysfunction to those of matched healthy volunteers. The acquisition data of normal subjects for both unreversed and reversed visually-guided tracking show clear similarities to the learning patterns generally reported in manual tracking paradigms and also observed by Frith and coworkers [8] in the study which formed the basis for the experimental set-up used in our investigation. The learning curves are characterised by improvements within the first minute of practice; this is followed by small increases of the time-on-target scores within the next two minutes. After a rest break, a large improvement in tracking performance occurs in the second relative to the first 3-min training session. Between-session increases in the time-on-target scores have been interpreted as evidence of increasing automatisation, more permanent learning or - in other terms - of the acquisition of a skill (see [8]). These effects were statistically significant for the control subjects in all analyses carried out for the different tracking measures.

As mentioned above, the tracking tasks were modelled on the procedure described by Frith et al. [8]. One procedural difference was the frequency of the horizontal target movement in the semi-predictable task which was halved in the present investigation in order to be able to include as many patients as possible (see Methods). This led to larger time-on-target scores for the vertical component in the control subjects, and to a lesser extent also in the PD patients compared to the Frith et al. study [8], whereas the scores for the horizontal direction were not markedly different. It is conceivable that subjects in the present study allocated more resources to tracking the vertical direction since the horizontal direction was slowed down and therefore less demanding. There were, however, no apparent ceiling effects, since significant improvements were observed in the control group despite high initial time-on-target scores. In the mirror-reversed condition, the overall performance of the control subjects was poorer than the scores reported in the Frith et al. study [8] for the horizontal direction. Whether this performance difference relates to minor differences in the experimental set-up (e.g., concerning the gain or the length of the joystick) cannot be answered conclusively.

The main purpose of this investigation was to analyse the motor learning pattern of patients with different types of motor system disorders, i.e., patients with striatal or cerebellar dysfunction. Both patient groups 
showed evidence of motor skill acquisition deficits compared to the learning curves of their matched control subjects, but the specific pattern of impairment was different. It may be argued that the general motor performance deficit observed in PD patients and patients with cerebellar dysfunction relative to control subjects (and also reported for PD by Frith et al. [8] on a similar tracking task) does not allow an interpretation of the learning data. However, the finding that both patient groups showed preserved learning relative to controls on some aspects of the tasks while being impaired on others indicates that a motor deficit alone cannot explain the more specific skill acquisition impairments. In addition, within-group analyses suggested that PD patients showed significant learning on the unreversed but not the mirror-reversed task, whereas the CERE patients tended to show the opposite pattern. This finding may be seen as evidence for dissociable difficulties in motor skill acquisition in the two patient groups.

PD patients consistently had problems in adaptation or acquisition of a motor set within the first minute of tracking. These results are consistent with the observations reported by Frith et al. [8] and they have been attributed to temporary problems in adapting existing motor programs to novel situations. As the time-ontarget scores of the PD patients increased to a very small degree or - in some instances - even decreased over the first minute of practice, it might be argued that the term 'adaptation' does not apply to the data of the PD patients. Instead, the change within the first minute might reflect changes in strategy that are made necessary by the motor disorder and - at least in case of the semi-predictable task - enabled the PD patients to improve their tracking performance over time. The degree of this improvement did not differ from the controls.

Deficits in the components of learning which represent skill acquisition were only observed for mirrorreversed tracking and therefore under conditions of more difficult visuo-motor coordination when opposing visual and proprioceptive informations had to be integrated. These findings share some similarities with previously published data on the involvement of the striatum in motor learning. A SPECT study demonstrated basal ganglia activation during a mirrorreversed tracking task which was conceptually similar to the paradigm used in the present study (in addition to SMA, frontal cortex and cerebellum were activated [17]). Frith et al. [8] reported smaller betweensession improvements in tracking performance for both unreversed and reversed conditions, but it is note- worthy that the data of only 1 PD patient had to be excluded from their analysis because of poor performance (less than $5 \%$ on time) on the unreversed task, while 4 patients had to be excluded for the same reason from the reversed task. This indicates that the mirrorreversed condition was particularly difficult for the PD patients.

There are several possible explanations for the skill acquisition impairment of PD patients during learning the reversed task. The PD patients may have been more susceptible to interference from the routine of unreversed tracking which always preceded the reversed tracking session (see [9]). There is, however, no clear evidence for this interpretation, since the patients were in the early stages of PD and did not show deficits on frontal tests (see [4]). The second explanation relates to the fact that the reversed condition placed relatively high demands on visuomotor processing and spatial reorganisation and therefore required a high degree of internal elaboration. Within the context of motor tasks, the supplementary motor area (SMA) appears to be implicated in such elaboration processes (see [17]), and a patient with an SMA lesion was recently shown to have problems with the acquisition of mirror-reversed, but not with unreversed tracking [1]. This clearly parallels the findings observed in the PD patients, and as the SMA and the basal ganglia are reciprocally connected, it is likely that circuits involving both structures are implicated in acquisition of tracking tasks which require a high degree of internal control.

Patients with cerebellar dysfunction showed a different pattern of motor learning problems. Significant impairments of skill acquisition in terms of reduced between-session improvements were only found during tracking of a regular and predictable movement. Patients with cerebellar damage thus appear to have problems in anticipating the target movements. This result cannot be explained by the lack of knowledge about the regularity of the movement since the proportion of subjects who noticed this feature was identical in the cerebellar patients and their control group. Deficits in learning to trace regular movements were also reported by Sanes and coworkers [21]. Patients with cerebellar disorders were impaired at the repetitive tracing of a geometric pattern under direct visual control; their performance for mirror-reversed tracing, however, did not differ from the data of the control subjects. Improvements in tracking a regular predictable component requires initiation of the movement before visual cues control the movement (see [8]). The results of the present study indicate that movement ini- 
tiation may be compromised in cerebellar disorders which might relate to bradykinesia (see [13, 21]).

Taken together, the present findings offer further empirical evidence for the hypothesis that both striatum and cerebellum are implicated in the acquisition and the storage of motor skills [6, 23]. Both areas form part of a neuronal network which involves cerebellum, thalamus, basal ganglia and the frontal cortex [22]. The specific contribution of each area depends upon the specific requirements and features of a motor learning task. The results of this investigation suggest that the striatum plays an important role during motor learning if a high degree of internal elaboration is involved in movement control whereas the cerebellar contribution may relate to the control of anticipatory movements.

\section{Acknowledgements}

This research was supported by the German Research Society (Deutsche Forschungs-gemeinschaft, Da 259/4-2).

\section{References}

[1] H. Ackermann, I. Daum, M.M. Schugens and W. Grodd, Impaired procedural learning after damage to the left supplementary motor area (SMA), Journal of Neurology, Neurosurgery, and Psychiatry 60 (1996), 94-97.

[2] I. Daum, H. Ackermann, M.M. Schugens, C. Reimold, J. Dichgans and N. Birbaumer, The cerebellum and cognitive functions in humans, Behavioral Neuroscience 107 (1993), 411-419.

[3] I. Daum, M.M. Schugens, H. Ackermann, W. Lutzenberger, J. Dichgans and N. Birbaumer, Classical conditioning after cerebellar lesions in humans, Behavioral Neuroscience $\mathbf{1 0 7}$ (1993), 748-756

[4] I. Daum, C. Reimold and S. Spieker, Kognitive Beeinträchtigungen im Frühstadium der Parkinsonschen Krankheit. Eine explorative Studie an 18 unmedizierten de novo Patienten, Zeitschrift für Gerontopsychologie und -psychiatrie 7 (1994), 85-94.

[5] I. Daum, M.M. Schugens, C. Breitenstein, H. Topka and S. Spieker, Classical eyeblink conditioning in Parkinson's disease, Movement Disorders 11 (1996), 639-646.

[6] J. Doyon, Skill learning, International Review of Neurobiology 41 (1997), 273-294

[7] J. Doyon, D. Gaudreau, R. Laforce and M. Castonguay, Role of the striatum, cerebellum, and frontal lobes in the learning of a visuomotor sequence, Brain and Cognition 34 (1997), $218-245$.
[8] C.D. Frith, C.A. Bloxham and K.N. Carpenter, Impairment in the learning and performance of a new manual skill in patients with Parkinson's disease, Journal of Neurology, Neurosurgery and Psychiatry 49 (1986), 661-668.

[9] C.D. Frith and R. Lang, Learning and reminiscence as a function of target predictability in a two-dimensional tracking task, Quarterly Journal of Experimental Psychology 31 (1979), 103-109.

[10] D.L. Harrington, K.Y. Haaland, R.A. Yeo and E. Marder, Procedural memory in Parkinson's disease: Impaired motor but not visuoperceptual learning, Journal of Clinical and Experimental Neuropsychology 12 (1990), 323-339.

[11] W.C. Heindel, D.P. Salmon, C.W. Shults, P.A. Walicke and N. Butters, Neuropsychological evidence for multiple implicit memory systems: A comparison of Alzheimer's, Huntington's and Parkinson's disease patients, Journal of Neuroscience 9 (1989), 582-587.

[12] M.M. Hoehn and M.D. Yahr, Parkinsonism: onset, progression, and mortality, Neurology 17 (1967), 427-442.

[13] G. Holmes, The symptoms of acute cerebellar injuries due to gunshot injuries, Brain 40 (1917), 461-535.

[14] G.M. Jackson, S.R. Jackson, J. Harrison, L. Henderson and C. Kennard, Serial reaction time learning and Parkinson's disease: Evidence for a procedural learning deficit, Neuropsychologia 5 (1995), 577-593.

[15] N. Jordan and H.J. Sagar, The role of the striatum in motor learning: Dissociations between isometric motor control processes in Parkinson's disease, International Journal of Neuroscience 77 (1994), 153-165.

[16] R. Lalonde and M.I. Botez, The cerebellum and learning processes in animals, Brain Research Review 15 (1990), 325332.

[17] W. Lang, M. Lang, I. Podreka, M. Steiner, F. Uhl, E. Suess, C. Müller and L. Deecke, DC-potential shifts and regional cerebral blood flow reveal frontal cortex involvement in human visuomotor learning, Experimental Brain Research $\mathbf{7 1}$ (1988), 353-364.

[18] D. Marr, A theory of cerebellar cortex, Journal of Physiology 202 (1969), 437-470.

[19] M. Mishkin, B. Malamut and J. Bachevalier, Memories and habits: Two neural systems, in: Neurobiology of learning and memory, G. Lynch, J.L. McGaugh and N.M. Weinberger, eds, Guilford Press, New York, 1984, pp. 65-77.

[20] A. Pascual-Leone, J. Grafmann, K. Clark, S. Stewart, J.S. Massaquoi, M. Lou and M. Hallett, Procedural learning in Parkinson's disease and cerebellar degeneration, Annals of Neurology 34 (1993), 594-602.

[21] J.N. Sanes, B. Dimitrov and M. Hallett, Motor learning in patients with cerebellar dysfunction, Brain 113 (1990), 103120.

[22] J.D. Schmahmann, An emerging concept: The cerebellar contribution to higher function, Archives of Neurology 48 (1991), 1178-1187.

[23] R.J. Seitz, P.E. Roland, C. Bohm, T. Greiz and S. StoneElander, Motor learning in man: a positron emission tomography study, NeuroReport 1 (1990), 57-66.

[24] L.R. Squire, Memory and the hippocampus. A synthesis from finding with rats, monkeys and humans, Psychological Review 99 (1992), 195-231. 


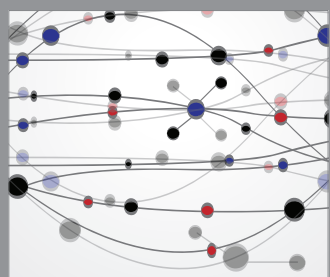

The Scientific World Journal
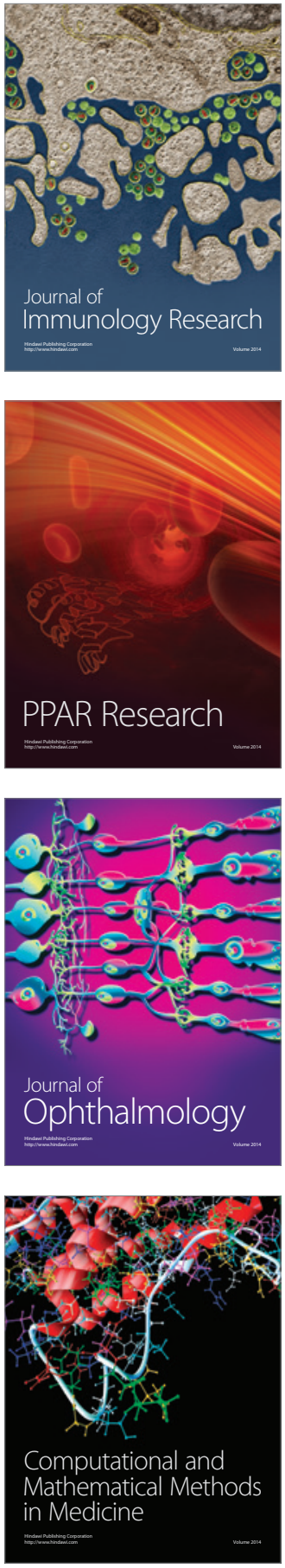

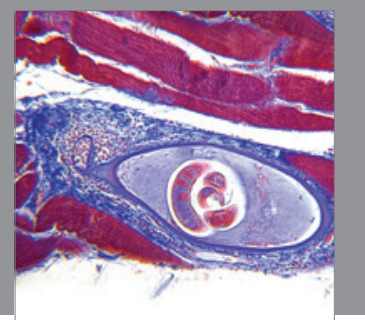

Gastroenterology

Research and Practice
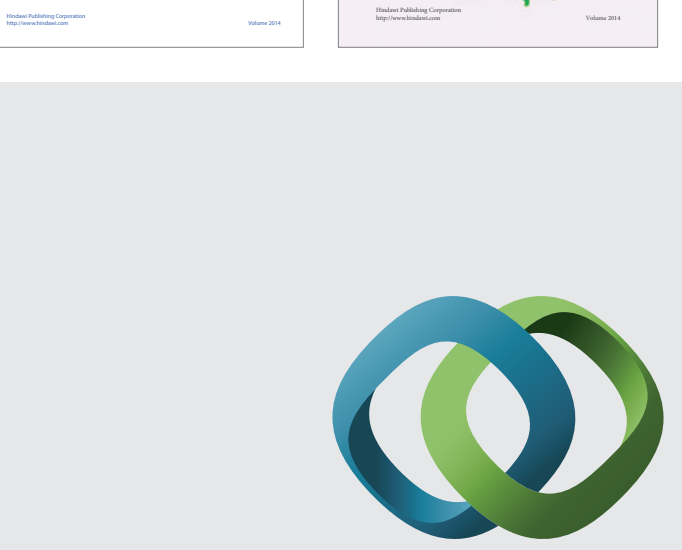

\section{Hindawi}

Submit your manuscripts at

http://www.hindawi.com
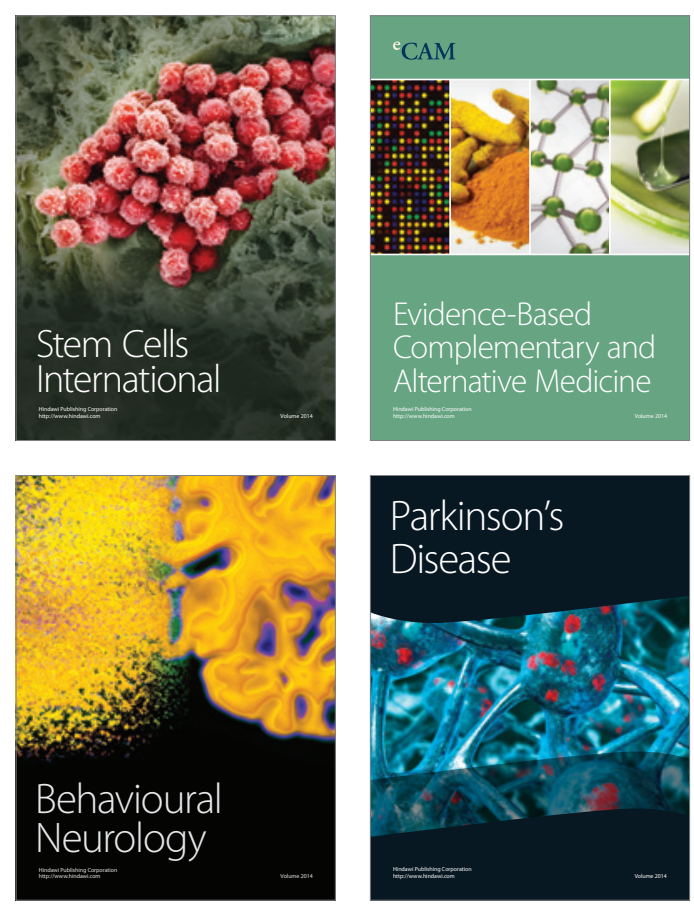

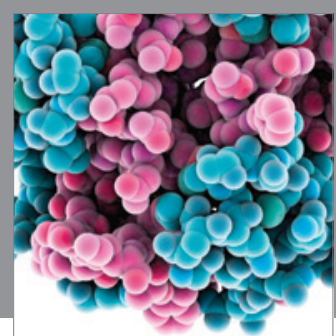

Journal of
Diabetes Research

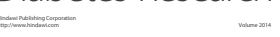

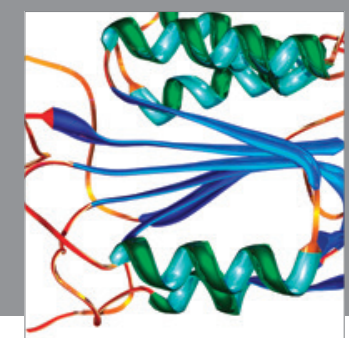

Disease Markers
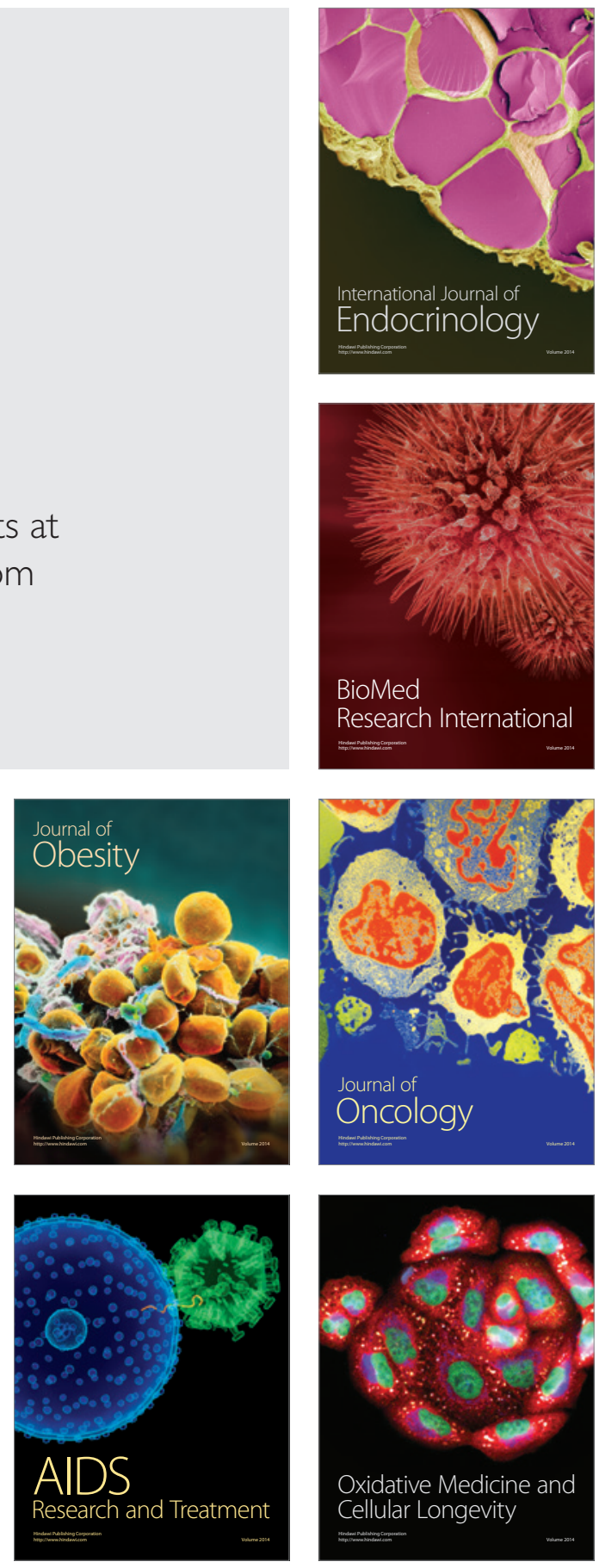